\title{
Rapid Mass Spectrometric Diagnosis of Bladder Cancer via Urine Carbonyl Metabolic Fingerprints
}

\section{Zongxiu Nie ( $\nabla$ znie@iccas.ac.cn )}

Institute of Chemistry Chinese Academy of Sciences

\section{Yuze Li}

Institute of Chemistry Chinese Academy of Sciences

\section{Lixia Jiang}

Department of Laboratory Medicine, First Affiliated Hospital of Gannan Medical University

\section{Zhenpeng Wang}

Institute of Chemistry, CAS

\section{Xiaohua Cao}

College of Chemical Engineering, Jiujiang University

\section{Lingwei Meng}

Institute of Chemistry Chinese Academy of Sciences

Jinghan Fan

University of Chinese Academy of Sciences

\section{Guanghui Wang}

Institute of Chemistry, Chinese Academy of Sciences

\section{Caiqiao Xiong}

Institute of Chemistry

\section{Article}

Keywords: bladder cancer (BC), carbonyl metabolic fingerprints, mass spectrometry

Posted Date: February 12th, 2021

DOI: https://doi.org/10.21203/rs.3.rs-199199/v1

License: (c) (1) This work is licensed under a Creative Commons Attribution 4.0 International License.

Read Full License 


\section{Rapid Mass Spectrometric Diagnosis of Bladder Cancer via Urine}

\section{Carbonyl Metabolic Fingerprints}

Yuze Li ${ }^{1,2}$, Lixia Jiang ${ }^{3}$, Zhenpeng Wang ${ }^{4}$, Xiaohua Cao ${ }^{5}$, Lingwei Meng ${ }^{1,2}$, Jinghan Fan ${ }^{2}$, Guanghui Wang ${ }^{4}$, Caiqiao Xiong ${ }^{1 *}$, and Zongxiu Nie ${ }^{1,2 *}$

${ }^{1}$ Beijing National Laboratory for Molecular Sciences, Key Laboratory of Analytical Chemistry for Living Biosystems, Institute of Chemistry, Chinese Academy of Sciences, Beijing, 100190,

China

${ }^{2}$ University of Chinese Academy of Sciences, Beijing, 100049, China

${ }^{3}$ Department of Laboratory Medicine, First Affiliated Hospital of Gannan Medical University, Ganzhou, Jiangxi Province, 341000, China

${ }^{4}$ National Center for Mass Spectrometry in Beijing, Beijing, 100190, China

${ }^{5}$ College of Chemical Engineering, Jiujiang University, Jiujiang, Jiangxi, 332005, China

*E-mail:xiongcq@iccas.ac.cn; znie@iccas.ac.cn 
Abstract: The diagnosis of bladder cancer (BC) is currently based on cystoscopy, which is invasive and expensive. Here, we described a non-invasive, low-cost $\mathrm{BC}$ diagnosis method based on a desorption, separation, and ionization mass spectrometry platform (DSI-MS) that adopts N, NDimethylethylenediamine (DMED) as a differential labeling reagent. The DSI-MS platform avoids the interferences from intra- and/or inter-samples, while the DMED increases detection sensitivity and distinguishes carboxyl, aldehyde, and ketone groups from untreated samples. Carbonyl metabolic fingerprints of urine from $28 \mathrm{BC}$ patients and 38 controls were portrayed and significant differences of some potential biomarkers were observed. The mechanisms of the changes have been discussed. Logistic regression (LR) was applied to discriminate BC from controls and an accuracy of $87 \%$ was achieved. We believe this patient-friendly method provides a hopeful approach for BC rapid point-of-care diagnostic.

\section{Introduction}

According to the World Cancer Research Fund, bladder cancer (BC) is the sixth most commonly occurring cancer in men and the 17 th in women with a high recurrence rate up to $50 \%-70 \%{ }^{1}$ Currently, BC diagnosis mainly relies on cystoscopy, a procedure in which a flexible or rigid tube bearing a camera is introduced into the bladder through the urethra. ${ }^{1}$ Although the diagnostic accuracy of this method is high, cystoscopy is painful, invasive, and expensive, ${ }^{2}$ making it unsuitable for point-of-care diagnostics ${ }^{3}$. Computed tomography (CT), magnetic resonance imaging (MRI), and other imaging methods also play significant roles in the management of patients with BC, but limited, to some extent, by interobserver variability, side effects of contrast agents, and expensiveness. ${ }^{4} \mathrm{~A}$ non-invasive, patient-friendly, cost-effective, and rapid diagnostic method for $\mathrm{BC}$ is urgently needed. $^{5}$

Bladder's cancerous may cause the slough of cancer cells in the surface, and contaminate the urine in it. ${ }^{6}$ Therefore, careful investigation of the composition of urine, such as metabolic small 
molecules can potentially provide a non-invasive urinary cancer/disease diagnosis $\operatorname{method}^{7}$. Mass spectrometry (MS) is a powerful method for metabolomics studies, and it has been used for investigating the relationship between $\mathrm{BC}$ and urine metabolites. ${ }^{8-12}$ Carbonyl (such as carboxyl, aldehyde, and ketone) compounds represent a kind of important metabolites in urine ( $40 \%$ urine metabolites are carbonyl compounds), and significant concentration changes of these compounds could be induced by BC. $8,9,13$ Coupling with chromatographic separation and principal component analysis (PCA), MS methods show the potential of accurate diagnosis of $\mathrm{BC}{ }^{11}$ However, conventional liquid or gas chromatographic separation is time-consuming. ${ }^{14,} 15$ Tedious sample pretreatment and column washing steps are inevitable. ${ }^{11}$ Ambient ionization $\mathrm{MS}^{16-19}$ attracts considerable attention recently for its' growing applications in clinical diagnosis. ${ }^{20-22}$ Various ambient ion sources were developed for point-of-care diagnosis of lung cancer, ${ }^{23}$ human glioma, ${ }^{24}$ oral squamous cell carcinoma, ${ }^{25}$ and strep throat ${ }^{26}$, etc. This exquisite technique simplifies sample pretreatment and shortens detection time, but lacks separation ability. Besides, the identification of isomers with different functional groups, such as carboxyl, aldehyde, and ketone in the complex sample is still challenging just based on the molecular weight determined by MS. ${ }^{27,28}$ Derivatization methods of specific functional group improve the sensitivity and selectivity of MS largely. ${ }^{29-33}$ However, simultaneous multiple functional groups derivatization with only one reagent for MS detection has been rarely reported.

To overcome these challenges, a rapid BC diagnosis method based on MS was established. Figure 1 shows the typical procedure of this method. The urine sample was first mixed with derivatization reagent $\mathrm{N}, \mathrm{N}$ - Dimethylethylenediamine (DMED), which can achieve the differential labeling of carbonyl groups simultaneously. Subsequently, $10 \mu \mathrm{L}$ of the mixture was loaded on a piece of sand core chip placed on a heated holder. With the increase of temperature (from $25^{\circ} \mathrm{C}$ to $250{ }^{\circ} \mathrm{C}$ ), the volatile metabolites in urine can be desorbed in order, and can be further ionized by an atmospheric pressure glow discharge (APGD) ion source. Here the combination of APGD, sand core chip, and 
heated holder was renamed as desorption, separation, and ionization mass spectrometry (DSI-MS) platform, and the details of the DSI-MS platform were shown in Figure S1. By analyzing the spectra and searching for the Human Metabolome Database (HMDB), these metabolites can be identified. Machine learning methods were then employed to classify the BC group and the healthy control (HC) group.

\section{Results and Discussion}

\section{The DSI-MS platform}

The angle between APGD and the horizontal line, the distance from the MS inlet to the APGD discharge region, the discharge current and gas flow rate were optimized to $45^{\circ}, 4 \mathrm{~mm}, 8 \mathrm{~mA}$ and 0.6 $\mathrm{L} / \mathrm{min}$ respectively (Figure S2). The sand core chips with different micropore diameters (G2, G3, G4, G5) were compared and G5 shows the best separation performance and will be used hereafter (Figure S3). To demonstrate the separation ability and the robustness of the DSI platform, a urine sample from a healthy volunteer was tested by loading it to the sand core chip directly. Figure 2a shows the extracted ion chromatogram (EIC) of several representative compounds in the urine. It can be seen that these compounds can be separated clearly. The corresponding retention times of each compound were listed in Table S1. The excess DMED was desorbed from the platform in the early time ( $\sim 0.1 \mathrm{~min}$.$) of the detection process, which facilitates the untargeted labeling of carbonyl$ compounds in urine with sufficient DMED. Similar to that in gas chromatography, the separation effect of the DSI can contribute to the porous inner structure of the sand core chips and the temperature increasing caused by heating. Compounds with higher volatile and lower adhesive force (van der Waals force) to the tiny cavity wall of the sand core chip will be desorbed earlier. When the sand core chip was removed, the separation effect would disappear (Figure 2b). The DSI platform shows good reproducibility between multiple loadings on one chip (relative standard deviation, $\mathrm{RSD}=5.97 \%)$ and between multiple chips $(\mathrm{RSD}=14.15 \%)$ as shown in Figure $\mathrm{S} 4$. The sand core chip 
is inexpensive ( $\$ 0.4$ per chip) and disposable, thus urine samples can be analyzed without pretreatment, and the intra- and/or inter-sample interferences can be avoided. In addition, the whole analysis time is less than 5 minutes (the derivatization reactions mentioned below require almost no more time), which is suitable for point-of-care diagnosis.

\section{The differential labeling strategy}

To identify carbonyl compounds in urine, derivatization reactions were performed by adding $2.5 \%$ (v:v) DMED into samples. Scheme 1 shows the predicted derivatization reaction and the fragmentation of the derivatives in collision induced dissociation (CID). Theoretically, these derivatives exist in pairs of isomers with different charge sites (Figure S5). The charge site can keep in either the tertiary amine nitrogen or the bridging nitrogen. The free energy of these isomers was calculated, and the isomer with lower energy in each pair was shown in Scheme 1. For the derivatives of carboxyl and ketone, the charge site would keep in the tertiary amine nitrogen, and the bridging nitrogen, respectively. Specially, for aldehyde compounds, since part of aldehydederivative can be further oxidized, they possess both the charging cases, where the charge site of the derivative is in the bridging nitrogen, and the charge site of the oxide is in the tertiary amine nitrogen. Charge site-initiated cleavage occurs in the $\mathrm{C}-\mathrm{N}$ bond nearing the charge site during the CID process (Figure S5). Thus the tertiary amine nitrogen-charged isomer generates daughter ion at $\Delta m / z=-45$ (type I fragmentation), while the bridging nitrogen-charged isomer generates daughter ion at $\mathrm{m} / \mathrm{z} 72$ (type II fragmentation). With respect to the low-energy isomer in each pair, carboxyl acid is featured by type I fragmentation, ketone is featured by type II fragmentation, and aldehyde is featured by both type I and type II fragmentations.

A series of compounds containing carbonyl groups were tested. The reactions take place rapidly induced by the increased temperature. Figure 3a-c show the mass spectra of benzoic acid, heptanone, and citronellal labeled by DMED. Their derivative peaks ( $\mathrm{m} / \mathrm{z} 193, \mathrm{~m} / \mathrm{z} 185$, and $\mathrm{m} / \mathrm{z} 225$ respectively) show an overwhelming advantage to their $[\mathrm{M}+\mathrm{H}]^{+}$peaks $(\mathrm{m} / \mathrm{z} 123,115$ and 155 , respectively), which 
indicates a high labeling efficiency. Unlike carboxyl and ketone, aldehyde-derivatives can be further oxidized $(\mathrm{m} / \mathrm{z} 241$, red dot noted in Figure 3c) due to a lower steric hindrance compared with ketone. The reactive oxygen species in the glow discharge provide the oxidant. ${ }^{34}$ The corresponding CID spectra of these derivatives are shown in Figure 3d-3g. The characteristic peak at $\Delta m / z=-45$ for benzoic $\operatorname{acid}^{35}$, peak at $m / z=72$ for heptanone, and both peaks at $\Delta m / z=-45$ and $m / z=72$ for citronellal were observed, which is consistent with the theoretical predictions. Similar rules have also been found in a series of other carbonyl compounds (Figure S6-S8). This method also shows the potential for simultaneous determination of the type and quantity of functional groups in the same molecule (Figure S9). The well-matched theoretical predictions and experimental outcomes show the reliability of this method.

\section{Analysis of clinical urine samples}

The labeling strategy and the DSI-MS platform would facilitate the identification of carbonyl metabolites in urine. The limit of detection (LOD) of this method was determined as $0.35 \mathrm{pmol} / \mathrm{L}$ for oleic acid (Figure S10a and b). Compared with underived oleic acid in negative ion mode (Figure S10c), tremendous enhancement of sensitivity was achieved. Figure 4a and b show the representative mass spectra of urine from BC and HC. Totally $28 \mathrm{BC}$ and $38 \mathrm{HC}$ samples (Figure 4c) were tested and repeated three times for each sample. In the $m / z$ range of 50-250, 37 acids, 4 ketones, and 1 aldehyde were detected and identified (Table S1). The relative quantification of the potential biomarkers in urine was achieved by using 9-phenylacridine, a stable exogenous compound, as an internal standard. Good linearity $\left(\mathrm{R}^{2}=0.988\right)$ between the ratio $\mathrm{R}(\mathrm{A} / \mathrm{I})$, i.e., the intensity of analytes divided by that of internal standard, and the concentration of the analyte was achieved (Figure S11). The logarithmic $\mathrm{R}(\mathrm{A} / \mathrm{I})$ of potential biomarkers were compared between $\mathrm{BC}$ vs. HC and shown in Figure 4d. Compared to HC, pyruvate, nicotinic acid, 4-hydroxybenzoic acid, glycolic acid, etc. show significant increases in BC. Meanwhile, ornithine, propionic acid, hippuric acid, hexanoic acid, etc. show significant decreases in BC $(p<0.05)$. 
Oxidation of pyruvate by cancer cells mitochondria would slow down and the oxidation of fatty acids would accelerate ${ }^{36}$. Such an effect benefits the growth of cancer cells. ${ }^{37}$ Consequently, the level of pyruvate, hydroxypyruvate would increase and the level of hexanoic acid would decrease, which is consistent with our experimental results (Figure 4d). The significant decrease of ornithine can attribute to the increased ornithine decarboxylase (ODC) level in the carcinoma cells. ODC is believed to be related to tumor-promoting activities of a variety of agents. ${ }^{38-40}$

\section{Machine learning for the classification of $\mathrm{BC} / \mathrm{HC}$}

The relative concentrations of those detected potential markers were set as features and the $\mathrm{BC} / \mathrm{HC}$ status were set as the targets for the classification by machine learning. The matrix consisted of features and targets shows visible differences between BC and HC (Figure S12). We compared the classification performance of machine learning based on several models. The results were listed in Table S2, and logistic regression (LR) performs best. PCA was also applied to clustering the data but with poor performance (Figure S13). Figure 5A shows the predicted BC probability of each sample (repeated three times) by LR. When the threshold is equal to 0.5 , a common setting, the accuracy score of the LR model was $85 \%$. Figure 5B shows the True Positive Rate (TPR) and False Positive Rate (FPR) versus threshold. To optimize the model, we switch the threshold from 0.5 to 0.26 , where the difference between TPR and FPR reaches the maximum, and the accuracy score increased to $87 \%$. The matrix was split into a training group (75\%) and a testing group (25\%), and a confusion matrix shown in Figure 5c visualized the testing results. To evaluate this model more comprehensively, 5-fold cross-validation (Figure S14) was performed, and the Receiver Operating Characteristic (ROC) curves are shown in Figure 5d. The average Area Under the Curve (AUC) reaches 0.84 , which indicates the valuable performance of the model. FreeViz optimization results show that hippuric acid, ornithine, perillic acid, heptanal, benzoic acid, etc. contribute to the classification significantly (Figure S15).

\section{Conclusions}


In conclusion, we developed a method for bladder cancer rapid diagnosis based on a desorption, separation, and ionization mass spectrometry (DSI-MS) platform and on-line derivatization of carboxyl, ketone, and aldehyde groups by N, N- Dimethylethylenediamine (DMED). By sequential desorption of urine components from the disposable sand core chips, the platform avoids the interand/or intra- sample interferences during the detection process. The DMED-labeling strategy increases detection sensitivity and provides vital structure information of unknown compounds. Urine samples from 38 healthy controls and 28 BC patients were tested and classified using a logistic regression model, and an accuracy of $87 \%$ was achieved. Dozens of potential biomarkers show significant changes in the BC group compared to controls. The metabolism mechanisms of the changes have also been partially discussed.

This method has the advantages of cost-effective, high-sensitivity, patient-friendly, and timesaving. Although further efforts may be required, we believe it provides a promising approach for the point-of-care diagnosis of bladder cancer.

\section{Materials and Methods}

Chemicals. All the carboxylic acid standards, the solvent dimethyl sulfoxide (DMSO), and the internal standard 9-phenylacridine were purchased from TCI (Tokyo, Japan), and all the ketones and aldehydes were purchased from J\&K Scientific Ltd. (Beijing, China). The labeling reagent N, NDimethylethylenediamine (DMED) was purchased from Sigma Aldrich (St. Louis, MO, USA). The water used in all experiments was prepared using a Milli-Q water purification system from Millipore (Milford, MA, USA). All standard solutions were prepared in a concentration of $10 \mathrm{mg} / \mathrm{mL}$ (for solids) or $10 \mu \mathrm{L} / \mathrm{mL}$ (for liquids), and $2.5 \%$ (volume) DMED was then added into the solutions. The internal standard 9-phenylacridine was added to the urine samples and the concentration is 1.5 $\mu \mathrm{mol} / \mathrm{mL}$. 
Instrumentation. Mass spectrometry (MS) experiments were mainly performed on a linear ion trap mass spectrometer (LTQ XL, Thermo Scientific, San Jose, CA). The LTQ capillary temperature was $300{ }^{\circ} \mathrm{C}$ and the tube lens voltage was $40 \mathrm{~V}$. A home-made desorption, separation, and ionization (DSI) platform was placed in front of the LTQ as shown in Figure S1. High-resolution MS was performed on an Impact HD Q-TOF mass spectrometer (Bruker Daltonics, Bremen, Germany), and a Fourier transform ion cyclotron resonance (FT-ICR) mass spectrometer (solariX, Bruker Daltonics, Germany). All the MS were operated in the positive ion mode.

The desorption, separation, and ionization (DSI) platform. An aluminum cylinder holder with a diameter of $20 \mathrm{~mm}$ and a height of $8 \mathrm{~mm}$ was drilled with two holes. An electric heater was placed in the first hole and a thermal resistor was placed in the second hole to heat and monitor the plate temperature. Upon the aluminum cylinder, the samples (typically $10 \mu \mathrm{L}$ ) were loaded to a disposable sand core chip, where the sample solution is temporarily stored due to its porous structure. Figure S1a and c show the schematic diagram and a photograph of the DSI platform. Complex samples such as urine can be desorbed and separated during the heating process and ionized by an atmospheric pressure glow discharge (APGD) ionization source (details are shown in Supporting Information Section 1.1).

Statistical analysis and calculation work. The raw data were processed by Thermo Xcalibur Qual Browser 4.0. Peaks information of $\mathrm{m} / \mathrm{z}$ and relative concentration were extracted to form aligned matrices for machine learning. The machine learning works based on several models were performed in Python 3.7 environment. The molecule energy data were calculated based on Density functional theory (DFT) at the B3LYP/6-31G(d,p) level of theory, using GAUSSIAN 09 software.

\section{Ethical Considerations}

All experiments were performed in accordance with relevant ethical regulations and approved by the Ethics Committee at First Affiliated Hospital of Gannan Medical University.

\section{Acknowledgements}


This work was supported by grants from the National Natural Sciences Foundation of China (Grant Nos. 21625504, and 21827807) and Chinese Academy of Sciences.

\section{Author contributions}

Z. N. initiated the project. Z. N., G. W., and C. X. designed the overall approach. Y. L. carried out all the experiments and wrote the manuscript. L. M. and X. C. carried out the calculation works. L. J. provided samples. J. F. analyzed the data. Z. W. helped with the construction of the DSI platform. All authors joined and discussed the manuscript.

\section{Competing financial interests}

The authors declare no competing financial interest.

\section{References}

(1) Kaufman, D. S.; Shipley, W. U.; Feldman, A. S., Bladder cancer. The Lancet 2009, 374 (9685), $239-249$.

(2) Svatek, R. S.; Hollenbeck, B. K.; Holmang, S.; Lee, R.; Kim, S. P.; Stenzl, A.; Lotan, Y., The economics of bladder cancer: costs and considerations of caring for this disease. Eur Urol 2014, 66 (2), 253-62.

(3) Cumberbatch, M. G. K.; Noon, A. P., Epidemiology, aetiology and screening of bladder cancer. Transl Androl Urol 2019, $8(1), 5-11$.

(4) Hafeez, S.; Huddart, R., Advances in bladder cancer imaging. BMC Med 2013, 11, 104.

(5) Tran, L.; Xiao, J. F.; Agarwal, N.; Duex, J. E.; Theodorescu, D., Advances in bladder cancer biology and therapy. Nat Rev Cancer 2021, 21 (2), 104-121.

(6) Lokeshwar, V. B.; Soloway, M. S., Current Bladder Tumor Tests: Does Their Projected Utility Fulfill Clinical Necessity? Journal of Urology 2001, 165 (4), 1067-1077.

(7) Yang, J.; Wang, R.; Huang, L.; Zhang, M.; Niu, J.; Bao, C.; Shen, N.; Dai, M.; Guo, Q.; Wang, Q.; Wang, Q.; Fu, Q.; Qian, K., Urine Metabolic Fingerprints Encode Subtypes of Kidney Diseases. Angewandte Chemie 2020, 59 (4), 1703-1710.

(8) Španěl, P.; Smith, D.; Holland, T. A.; Singary, W. A.; Elder, J. B., Analysis of formaldehyde in the headspace of urine from bladder and prostate cancer patients using selected ion flow tube mass spectrometry. Rapid Communications in Mass Spectrometry 1999, 13 (14), 1354-1359.

(9) Cauchi, M.; Weber, C. M.; Bolt, B. J.; Spratt, P. B.; Bessant, C.; Turner, D. C.; Willis, C. M.; Britton, L. E.; Turner, C.; Morgan, G., Evaluation of gas chromatography mass spectrometry and pattern recognition for the identification of bladder cancer from urine headspace. Analytical Methods 2016, 8 (20), 4037-4046.

(10) Rodrigues, D.; Pinto, J.; Araujo, A. M.; Monteiro-Reis, S.; Jeronimo, C.; Henrique, R.; de Lourdes Bastos, M.; de Pinho, P. G.; Carvalho, M., Volatile metabolomic signature of bladder cancer cell lines based on gas chromatography-mass spectrometry. Metabolomics 2018, 14 (5), 62.

(11) Issaq, H. J.; Nativ, O.; Waybright, T.; Luke, B.; Veenstra, T. D.; Issaq, E. J.; Kravstov, A.; Mullerad, M., Detection of bladder cancer in human urine by metabolomic profiling using high performance liquid chromatography/mass spectrometry. J Urol 2008, 179 (6), 2422-6.

(12) Danielsson, R.; Allard, E.; Sjöberg, P. J. R.; Bergquist, J., Exploring liquid chromatography-mass spectrometry fingerprints of urine samples from patients with prostate or urinary bladder cancer. Chemometrics and Intelligent Laboratory Systems 2011, 108 (1), 33-48. 
(13) Kim, J.-W.; Lee, G.; Moon, S.-M.; Park, M.-J.; Hong, S. K.; Ahn, Y.-H.; Kim, K.-R.; Paik, M.-J., Metabolomic screening and star pattern recognition by urinary amino acid profile analysis from bladder cancer patients. Metabolomics 2010, 6 (2), 202-206.

(14) Guo, K.; Li, L., High-performance isotope labeling for profiling carboxylic acid-containing metabolites in biofluids by mass spectrometry. Analytical chemistry 2010, 82 (21), 8789-93.

(15) Leng, J.; Wang, H.; Zhang, L.; Zhang, J.; Wang, H.; Guo, Y., A highly sensitive isotope-coded derivatization method and its application for the mass spectrometric analysis of analytes containing the carboxyl group. Anal Chim Acta 2013, 758, 114-21.

(16) Cooks, R. G.; Ouyang, Z.; Takats, Z.; Wiseman, J. M., Detection Technologies. Ambient mass spectrometry. Science 2006, 311 (5767), 1566-70.

(17) Takats, Z.; Wiseman, J. M.; Gologan, B.; Cooks, R. G., Mass spectrometry sampling under ambient conditions with desorption electrospray ionization. Science 2004, 306 (5695), 471-3.

(18) Wang, H.; Liu, J.; Cooks, R. G.; Ouyang, Z., Paper spray for direct analysis of complex mixtures using mass spectrometry. Angewandte Chemie 2010, 49 (5), 877-80.

(19) Sleeman, R.; Burton, I. F. A.; Carter, J. F.; Roberts, D. J., Rapid screening of banknotes for the presence of controlled substances by thermal desorption atmospheric pressure chemical ionisation tandem mass spectrometry. The Analyst 1999, 124 (2), 103-108.

(20) Casas-Ferreira, A. M.; Nogal-Sanchez, M. D.; Perez-Pavon, J. L.; Moreno-Cordero, B., Non-separative mass spectrometry methods for non-invasive medical diagnostics based on volatile organic compounds: A review. Anal Chim Acta 2019, 1045, 10-22.

(21) Ifa, D. R.; Eberlin, L. S., Ambient Ionization Mass Spectrometry for Cancer Diagnosis and Surgical Margin Evaluation. Clinical chemistry 2016, 62 (1), 111-23.

(22) Brown, H. M.; Pirro, V.; Cooks, R. G., From DESI to the MasSpec Pen: Ambient Ionization Mass Spectrometry for Tissue Analysis and Intrasurgical Cancer Diagnosis. Clinical chemistry 2018, 64 (4), 628-630.

(23) Lu, H.; Zhang, H.; Wei, Y.; Chen, H., Ambient mass spectrometry for the molecular diagnosis of lung cancer. The Analyst 2020, 145 (2), 313-320.

(24) Zou, R.; Cao, W.; Chong, L.; Hua, W.; Xu, H.; Mao, Y.; Page, J.; Shi, R.; Xia, Y.; Hu, T. Y.; Zhang, W.; Ouyang, Z., Point-of-Care Tissue Analysis Using Miniature Mass Spectrometer. Analytical chemistry 2019, 91 (1), 1157-1163.

(25) Song, X.; Yang, X.; Narayanan, R.; Shankar, V.; Ethiraj, S.; Wang, X.; Duan, N.; Ni, Y. H.; Hu, Q.; Zare, R. N., Oral squamous cell carcinoma diagnosed from saliva metabolic profiling. Proceedings of the National Academy of Sciences of the United States of America 2020.

(26) Jarmusch, A. K.; Pirro, V.; Kerian, K. S.; Cooks, R. G., Detection of strep throat causing bacterium directly from medical swabs by touch spray-mass spectrometry. The Analyst 2014, 139 (19), 4785-9.

(27) Ghaste, M.; Mistrik, R.; Shulaev, V., Applications of Fourier Transform Ion Cyclotron Resonance (FT-ICR) and Orbitrap Based High Resolution Mass Spectrometry in Metabolomics and Lipidomics. Int J Mol Sci 2016, 17 (6).

(28) Rathahao-Paris, E.; Alves, S.; Junot, C.; Tabet, J.-C., High resolution mass spectrometry for structural identification of metabolites in metabolomics. Metabolomics 2015, 12 (1).

(29) Shariatgorji, M.; Nilsson, A.; Fridjonsdottir, E.; Vallianatou, T.; Kallback, P.; Katan, L.; Savmarker, J.; Mantas, I.; Zhang, X.; Bezard, E.; Svenningsson, P.; Odell, L. R.; Andren, P. E., Comprehensive mapping of neurotransmitter networks by MALDI-MS imaging. Nature methods 2019, 16 (10), 1021-1028.

(30) Zhang, T.-Y.; Li, S.; Zhu, Q.-F.; Wang, Q.; Hussain, D.; Feng, Y.-Q., Derivatization for liquid chromatographyelectrospray ionization-mass spectrometry analysis of small-molecular weight compounds. TrAC Trends in Analytical Chemistry 2019, 119.

(31) Stadlmeier, M.; Bogena, J.; Wallner, M.; Wuhr, M.; Carell, T., A Sulfoxide-Based Isobaric Labelling Reagent for Accurate Quantitative Mass Spectrometry. Angewandte Chemie 2018, 57 (11), 2958-2962.

(32) Wang, M.; Han, R. H.; Han, X., Fatty acidomics: global analysis of lipid species containing a carboxyl group with a charge-remote fragmentation-assisted approach. Analytical chemistry 2013, 85 (19), 9312-20.

(33) Huang, T.; Armbruster, M. R.; Coulton, J. B.; Edwards, J. L., Chemical Tagging in Mass Spectrometry for Systems Biology. Analytical chemistry 2019, 91 (1), 109-125.

(34) Xie, X.; Wang, Z.; Li, Y.; Zhan, L.; Nie, Z., Investigation and Applications of In-Source Oxidation in Liquid Sampling-Atmospheric Pressure Afterglow Microplasma Ionization (LS-APAG) Source. Journal of the American Society for Mass Spectrometry 2017, 28 (6), 1036-1047.

(35) Zhu, Q. F.; Zhang, Z.; Liu, P.; Zheng, S. J.; Peng, K.; Deng, Q. Y.; Zheng, F.; Yuan, B. F.; Feng, Y. Q., Analysis of liposoluble carboxylic acids metabolome in human serum by stable isotope labeling coupled with liquid chromatography-mass spectrometry. J Chromatogr A 2016, 1460, 100-9.

(36) Samudio, I.; Fiegl, M.; Andreeff, M., Mitochondrial uncoupling and the Warburg effect: molecular basis for the reprogramming of cancer cell metabolism. Cancer Res 2009, 69 (6), 2163-6. 
(37) Lao-On, U.; Attwood, P. V.; Jitrapakdee, S., Roles of pyruvate carboxylase in human diseases: from diabetes to cancers and infection. J Mol Med (Berl) 2018, 96 (3-4), 237-247.

(38) H. Kanitz, E. I. L.; P. A. Schu, M., Investigation of Ornithine Decarboxylase Activity and Two-Dimensional Electrophoretic Protein Profile Following Exposure of T24 Bladder Carcinoma Cells to Tumor Promoter and Carcinogen. Toxicology Methods 2008, 7 (1), 27-41.

(39) TG, O. B., The induction of ornithine decarboxy lase as an early possibly obligatory event in mouse skin carcinogenesis. Cancer Research 1976, 36.

(40) Luk, G. D.; Hamilton, S. R.; Yang, P.; Smith, J. A.; O'Ceallaigh, D.; McAvinchey, D.; Hyland, J., Kinetic changes in mucosal ornithine decarboxylase activity during azoxymethane-induced colonic carcinogenesis in the rat. Cancer Res 1986, 46 (9), 4449-52. 


\section{Figures}

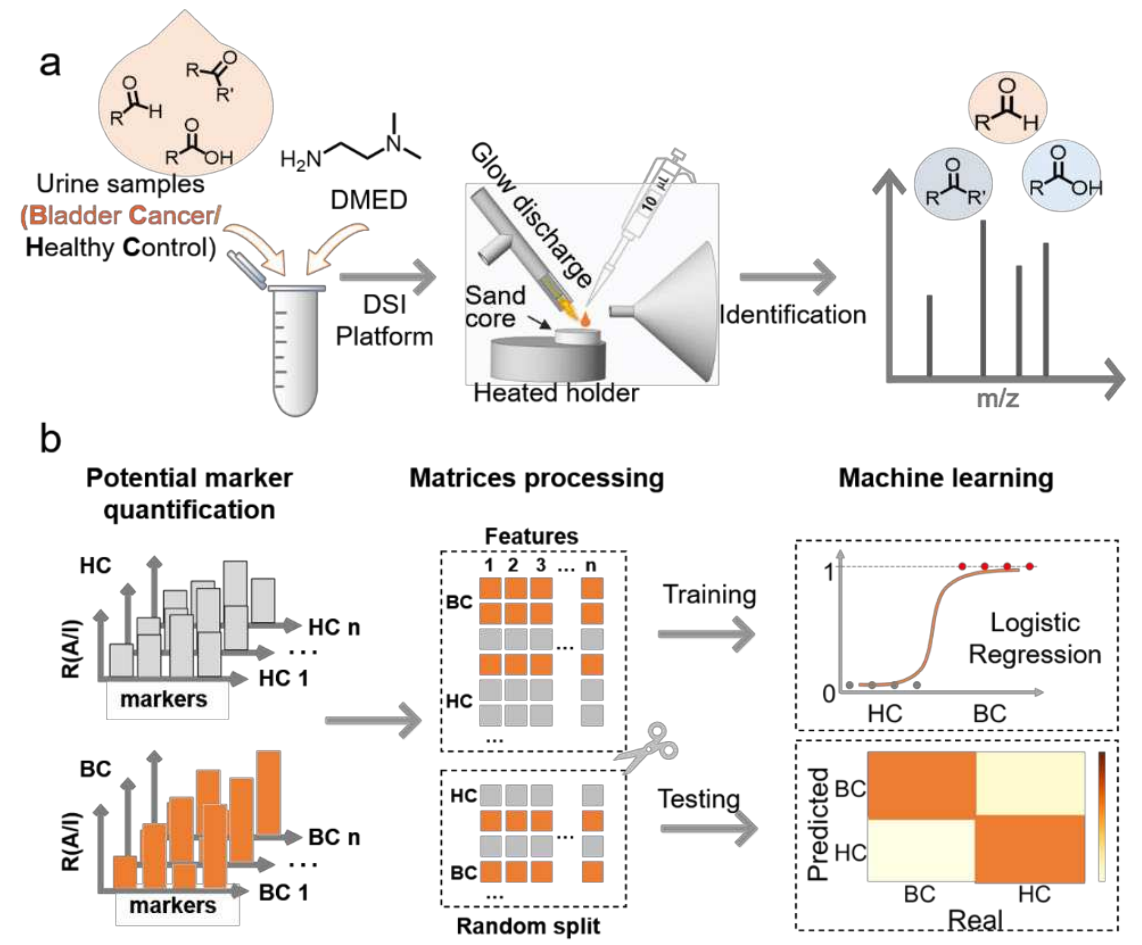

Figure 1. The workflow of the bladder cancer rapid diagnosis method established in this work. (a) By loading the mixture of untreated urine and DMED to the DSI-MS platform, carboxylic, ketonic, and aldehydic metabolites can be distinguished. (b) The relative concentrations of the identified potential markers were set as features and the $\mathrm{BC} / \mathrm{HC}$ status as the targe to form the matrix, and then the matrix was split into two parts randomly for the training and testing of the logistic regression model. 

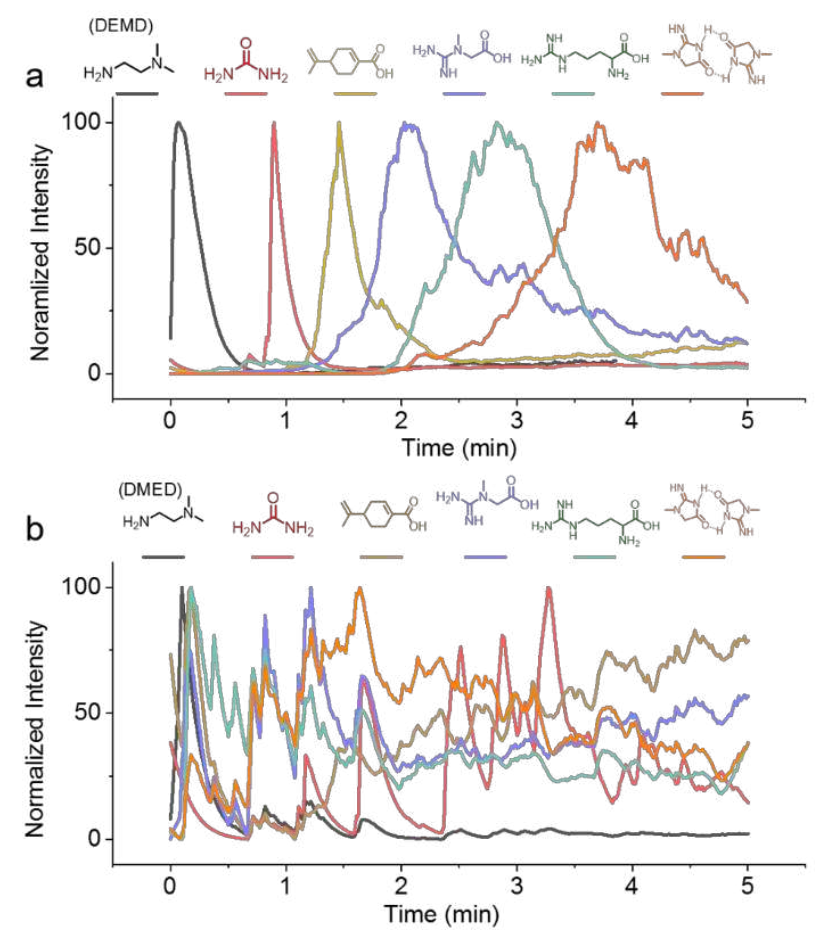

Figure 2. EIC of several representative compounds in urine samples separated by the DSI platform with (a) and without (b) the sand core chip. In the case of without the chip, the sample was loaded on the heated holder. 


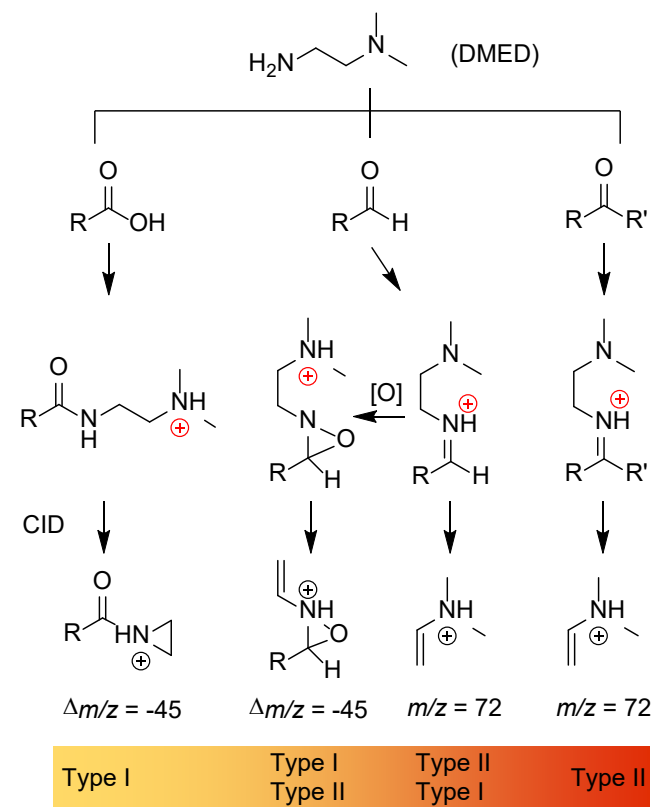

Scheme 1. The proposed pathway of carboxyl, aldehyde, and ketone labeled by DMED detected in MS1 and MS2 (CID). 


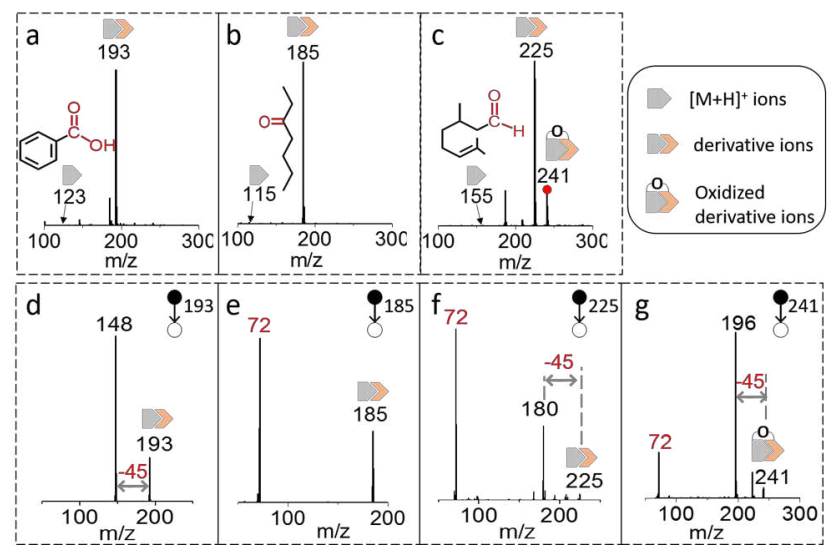

Figure 3. Mass spectra of (a) benzoic acid, (b) heptanone, and (c) citronellal labeled by DMED. The corresponding CID spectra of the derivatives are shown in (d), (e), and (f) respectively. (g) shows the CID spectrum of the oxidized derivative of citronellal. 


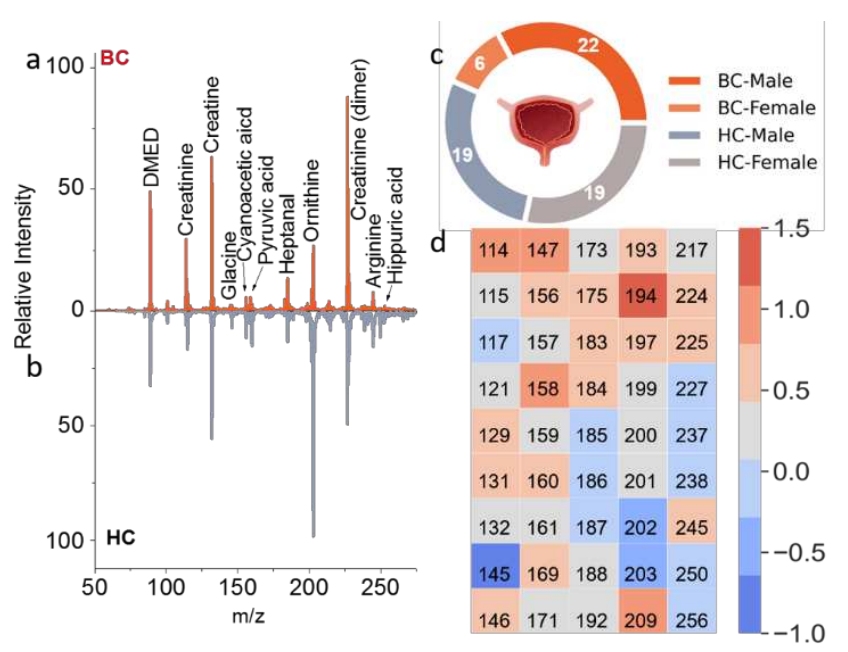

Figure 4. Average mass spectra of urine samples from (a) BC (b) HC. (c) Samples from 28 BC and $38 \mathrm{HC}$ were tested. (d) The heatmap shows the upregulation (red) or downregulation (blue) of these metabolites. (Numbers are the nominal $\mathrm{m} / \mathrm{z}$ of the corresponding metabolites). 

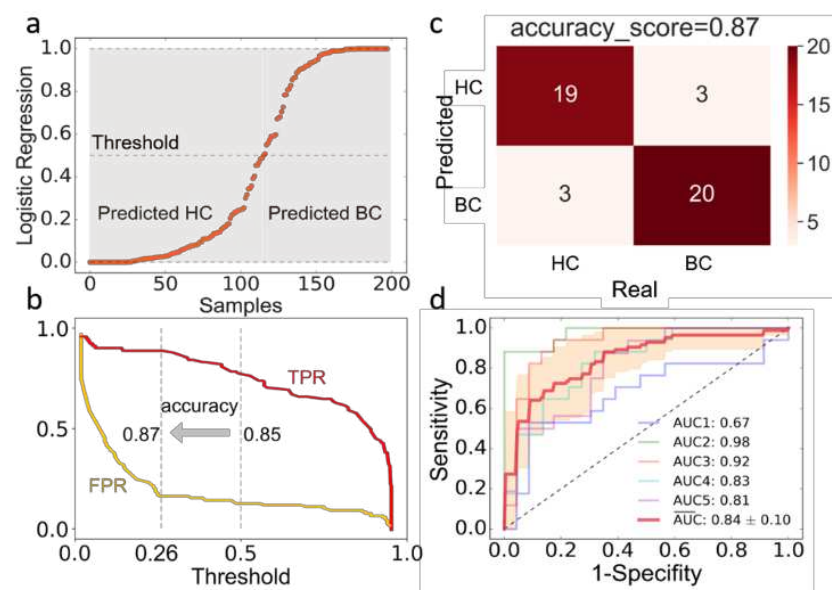

Figure 5. (a) Predicted BC probability of each sample (three-times repeated) by LR model. (b) The

True Positive Rate (TPR) and False Positive Rate (FPR) versus threshold. (c) Confusion matrix of the testing results of the random split dataset (training: testing $=3: 1$ ). (d) 5-fold cross-validation of the logistic regression model. 


\section{Figures}

a

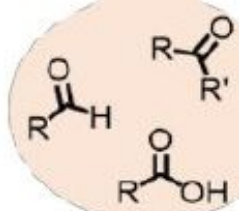

Urine samples

(Bladder Cancerl

Healthy Control)

b

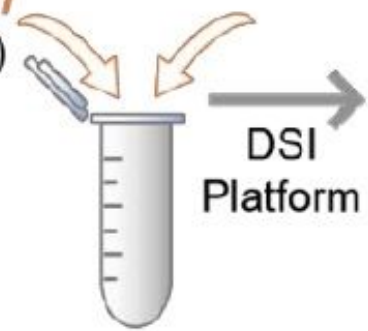<smiles>CN(C)CCN</smiles>

DMED
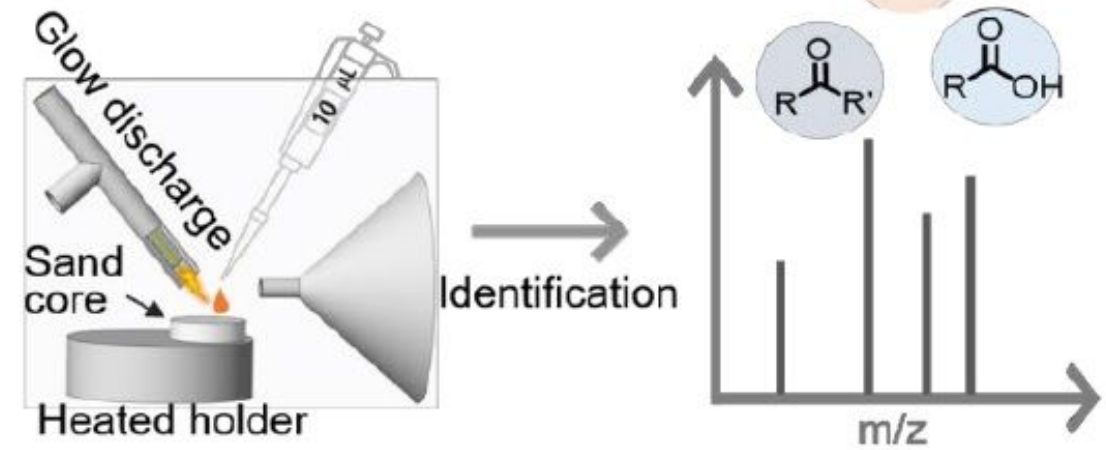

Potential marker quantification
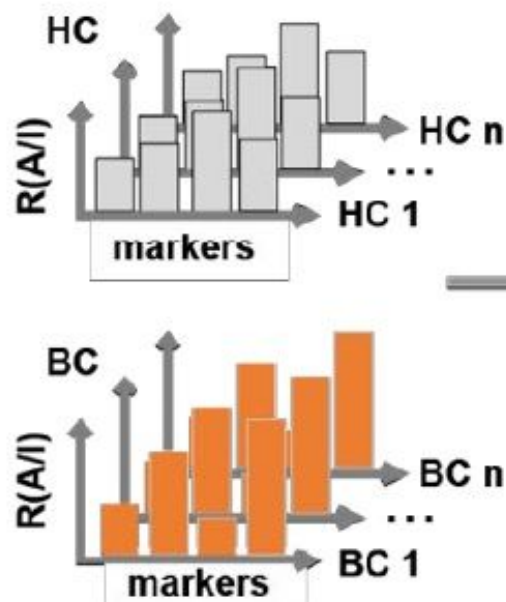

Matrices processing

Features

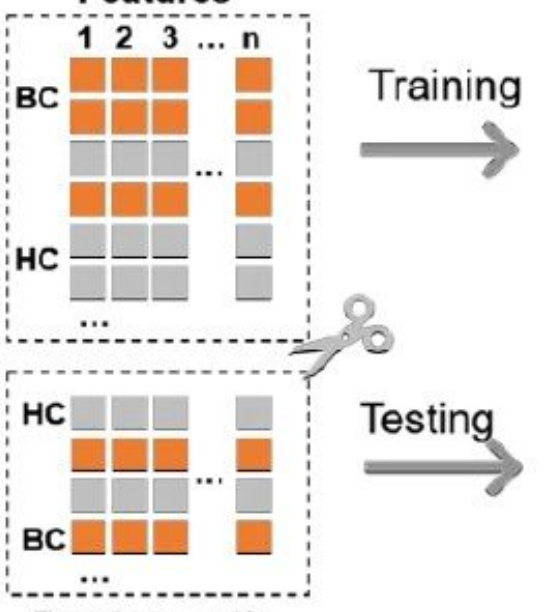

Random split
Machine learning

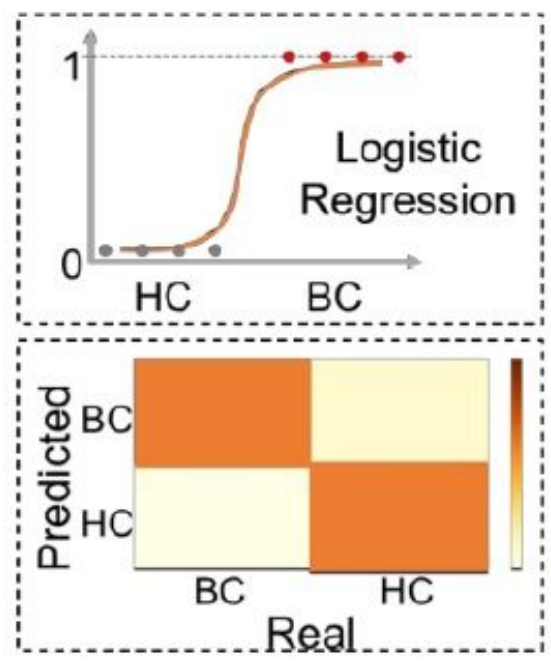

Figure 1

The workflow of the bladder cancer rapid diagnosis method established in this work. (a) By loading the mixture of untreated urine and DMED to the DSI-MS platform, carboxylic, ketonic, and aldehydic metabolites can be distinguished. (b) The relative concentrations of the identified potential markers were set as features and the $\mathrm{BC} / \mathrm{HC}$ status as the targe to form the matrix, and then the matrix was split into two parts randomly for the training and testing of the logistic regression model. 

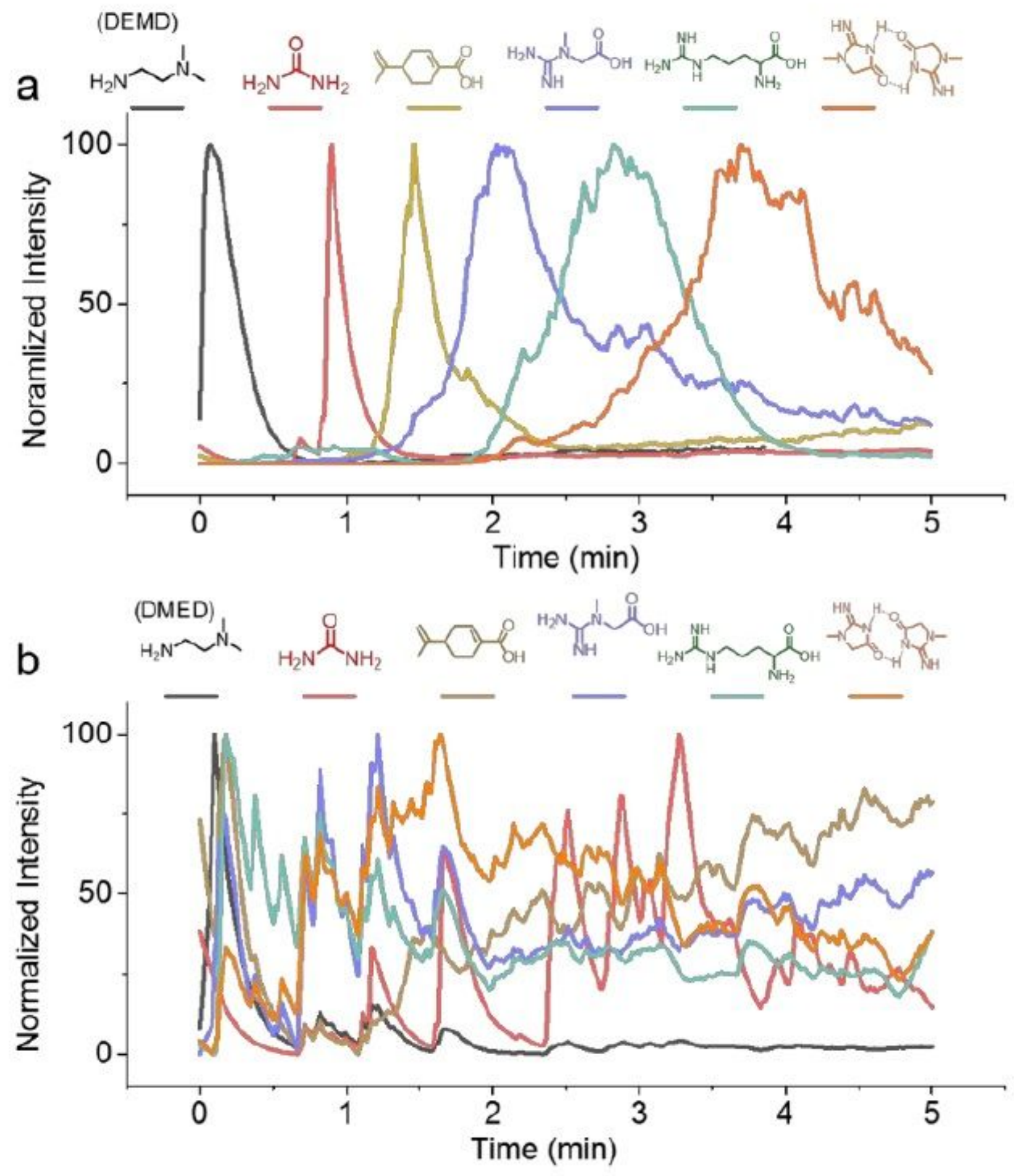

Figure 2

EIC of several representative compounds in urine samples separated by the DSI platform with (a) and without (b) the sand core chip. In the case of without the chip, the sample was loaded on the heated holder. 


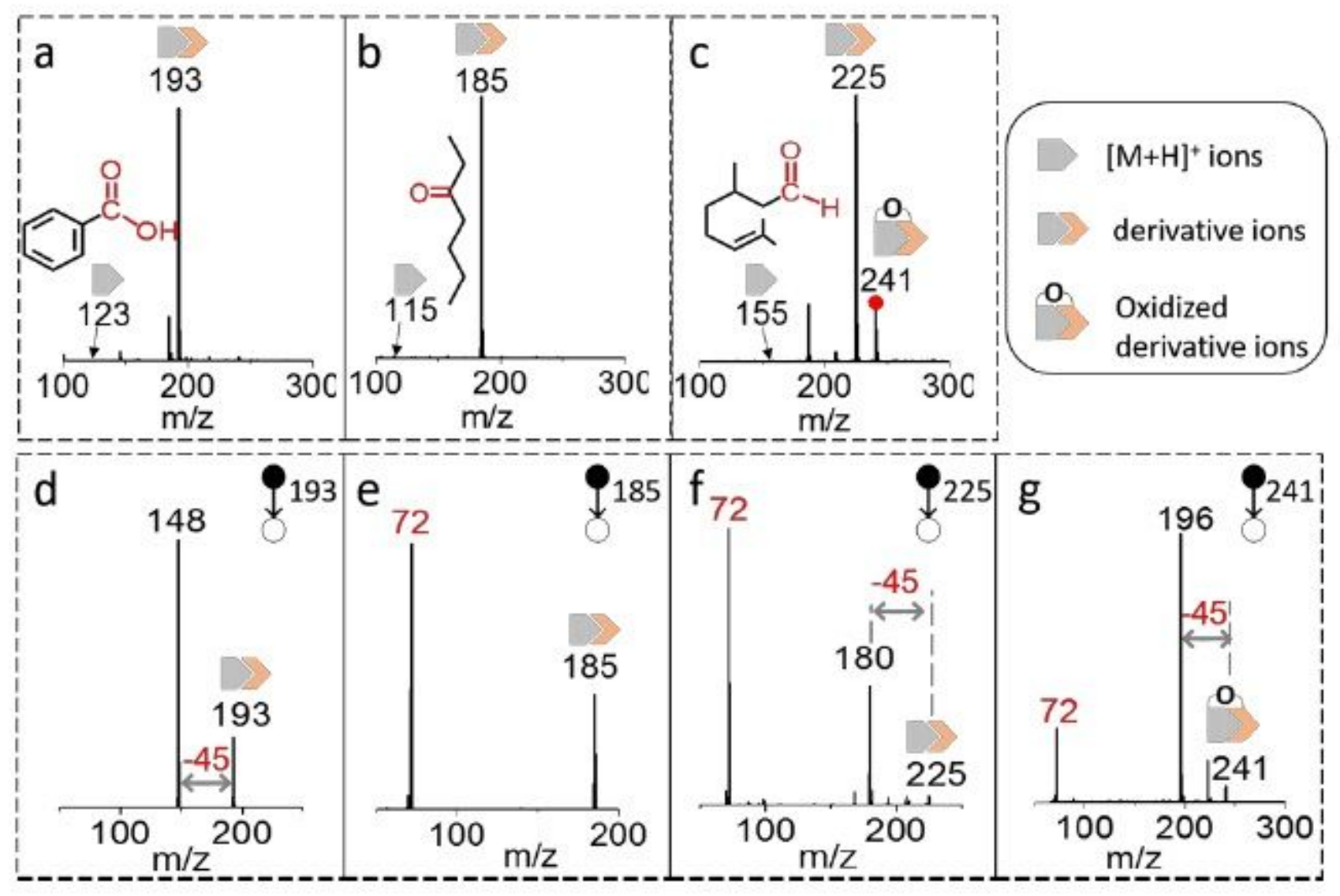

Figure 3

Mass spectra of (a) benzoic acid, (b) heptanone, and (c) citronellal labeled by DMED. The corresponding CID spectra of the derivatives are shown in (d), (e), and (f) respectively. (g) shows the CID spectrum of the oxidized derivative of citronellal. 


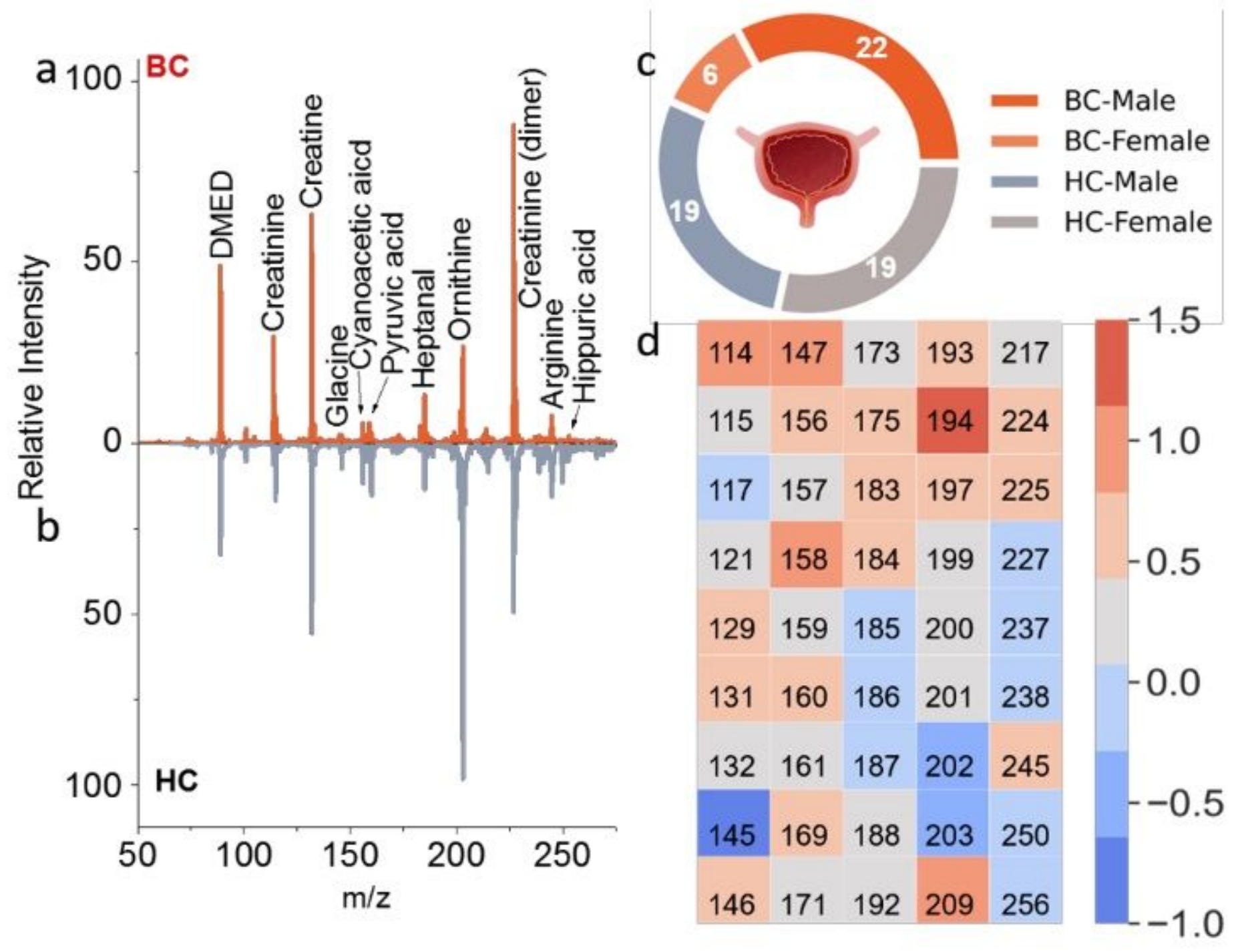

Figure 4

Average mass spectra of urine samples from (a) BC (b) HC. (c) Samples from 28 BC and $38 \mathrm{HC}$ were tested. (d) The heatmap shows the upregulation (red) or downregulation (blue) of these metabolites. (Numbers are the nominal $\mathrm{m} / \mathrm{z}$ of the corresponding metabolites). 

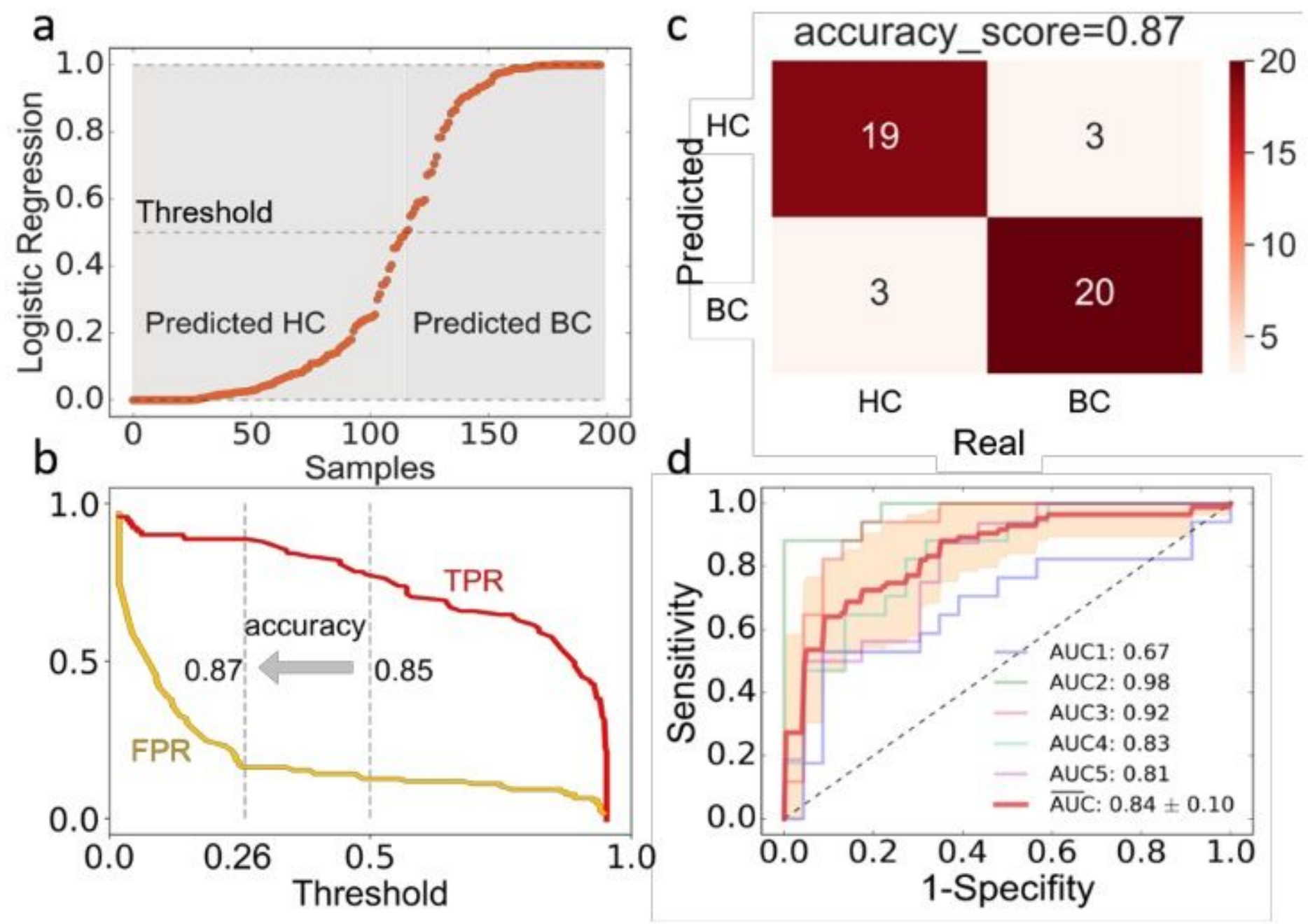

Figure 5

(a) Predicted BC probability of each sample (three-times repeated) by LR model. (b) The True Positive Rate (TPR) and False Positive Rate (FPR) versus threshold. (c) Confusion matrix of the testing results of the random split dataset (training: testing $=3: 1$ ). (d) 5 -fold cross-validation of the logistic regression model.

\section{Supplementary Files}

This is a list of supplementary files associated with this preprint. Click to download.

- SI.pdf 